\title{
Fatal Pulmonary Fat Embolism Occurring in Lumbar Spinal Fusion Surgery: Case Report and Review of Literature
}

\author{
Lu Zhang, Jie Zhang, Zihui Cheng, Lingjie Kong, Liang Wang, Long Yu, Songyue He \\ Beijing Source of Judicial Identification Center of Scientific Evidence, Beijing, China \\ DOI: $10.32629 / \mathrm{jcmr} . v 2 \mathrm{i} 4.551$
}

\begin{abstract}
Fat embolism system is one of the serious complications of orthopedic surgery, which is common seen in cases of severe trauma with long bone fractures. However, in clinical medical practice, it is rarely seen for Fat embolism system in orthopaedic lumbar fusion surgery. This paper report a case of sudden shock and death during lumbar fusion due to lumbar intervertebral disc disease. By forensic pathological examination, the pulmonary and brain fat embolism were observed in tissue sections, and the patient was diagnosed as FES. Based on the review of relevant literature and the pathological findings of this case, the clinical characteristics, diagnosis and treatment of FES in lumbar fusion are commentated in this paper. At the same time, it is emphasized that the clinical practise should strengthened the understanding and attention of non-traumatic fat embolism cases in order to timely identify, diagnose and treat the disease, improve the effectiveness of treatment, and reduce the corresponding medical disputes.
\end{abstract}

Keywords: lumbar spine, surgery, fat embolism, death, forensic pathology, judicial expertise

\section{Introduction}

Fat embolism (FE) was defined as the appearance of fat droplets in the small blood vessels of the patient's lungs, regardless of the presence or absence of the corresponding clinical manifestations. Fat embolism syndrome (FES) refers to a series of histopathological changes and clinical manifestations that occur from 10 to $40 \mathrm{~m}$ diameter fat particles that block blood vessels, organs, and tissues, especially the capillaries in the lungs. Due to onset of FES, the mortality rate is as high as $10 \% \sim 25 \%$, especially the case fatality rate of fulminant patients can be higher than $50 \%$. FES is not only the difficulty in clinical medical diagnosis and treatment, but also a difficulty in forensic medicine ${ }^{[1]}$. A large number of clinical studies and practices have demonstrated that FES is frequently discovered in bone trauma, especially in closed long bone fractures of lower limbs and in femur. However, FES reports related to spinal fusion are rarely reported in literature, and there is still little recognition of this in clinical practice. The author encountered a case of blood pressure declined suddenly and decreased heart rate during the operation of the spine and lumbar vertebrae. The patient died ineffective after clinical rescue, and pulmonary and brain fat embolism were confirmed by forensic autopsy and pathological examination. In order to arouse the attention of clinical colleagues and improve the ability of diagnosis and treatment, this paper reports and discussed combined with literature review.

\section{Cases introduction}

The patient, a 56-year-old male, is accompanied by pain in the right lower limb, intermittent claudication for 2 years, aggravated two months, and he was in terrible condition and admitted to hospital on Sep. 27, 2020. The previous patient had been suffering from "rheumatoid arthritis" for 6 years. During the diagnosis and treatment, lumbar MRI examination was performed, which showed L3/4 of the lumbar disc herniation, spinal canal stenosis, and anterior slippage of the third lumbar vertebra. Clinical diagnosis: Lumbar disc herniation and spondylolisthesis. To seek systematic treatment of operative surgery, the patient was admitted to the operating room for posterior lumbar intervertebral fusion in lumbar spine (L3/4) under general anesthesia at 14:00 PM, Sep. 30, 2020. Surgery performed a long posterior median incision of about $10 \mathrm{~cm}$ centered on the L3/4 spike gap. During the operation, the right inferior articular process of the third lumbar spine and the superior articular process of the fourth lumbar spine were chiselled, the lower part of the L3 vertebral body was gnawed, and the disc was resected. During the fusion operation, the removed bone was processed into granules and filled in the intervertebral space and the intervertebral fusion device was placed in room L3/4. As a result, the operation was smoothly conducted. However, when preparing suture (16:30pm), the patient suddenly experienced a drop in blood pressure and slow heart rate. The operation was immediately stopped and rescue measures such as continuous chest compressions, multiple intravenous injections of epinephrine and norepinephrine were taken. However, the patient's heart rate and blood pressure 
saw no sign of recovery, so rescue was continued and a second blood gas analysis was performed. The results showed that hemoglobin was $62 \mathrm{~g} / \mathrm{L}$ and $73 \mathrm{~g} / \mathrm{L}$, respectively. During the operation, the transfusion was returned after bleeding treatment, and transfused withred blood cell suspension $4 \mathrm{U}$ and plasma $400 \mathrm{ml}$. Electrical defibrillation was given at 17:46PM, but the heartbeat was not recovered and clinical death was declared one and a half hours later. Clinical cause of death: cardiac arrest.

After the death, the patient was performed by forensic pathological examination, no abnormal morphology of tissues and organs in the chest cavity and abdomen and pelvic cavity, but fractures of the left and right ribs 2,3,4,5 and sternum were observed as Cardiopulmonary Resuscitation. The surface of both lungs proved to be smooth, the cut surface was gray red, and the texture was soft. No foreign body was found in the pulmonary artery. No obvious bleeding was found in the abdominal cavity and pelvic cavity. The heart was $440 \mathrm{~g}$, the wall thickness of the left ventricle was $1.6 \mathrm{~cm}$, the wall thickness of the right ventricle was $0.3 \mathrm{~cm}$, no obvious abnormality was observed in each valve, the ventricle section was gray red, tough and no necrotic scar tissue was observed. Moderate stenosis of anterior descending coronary artery (grade II), no obvious stenosis of other coronary arteries. Microscopic observation showed that the alveolar structure of lung tissue was clear, some alveolar cavities contained edema fluid, and the interalveolar capillaries were dilated and congested. Some arterioles contain vacuoles of lipid droplets and a large number of small lipid droplets gathering together. Many white blood cells, cellulose and platelets were observed around the lipid droplets. Microthrombus formed by aggregation of leukocytes, fibrin and platelets can be seen in most small blood vessels of lung tissue. By using oil red special staining, many pulmonary alveolar wall capillaries and arterioles contained orange-red lipid droplets, and part of orange-red lipid droplets spilled out of blood vessels. No fresh ischemic necrotic lesions were found in the myocardium, with turbidities of myocardium cells, orderly arrangement of myocardial fibers and interstitial edema. The sinoatrial node and atrioventricular node are characterized by a large amount of fat infiltration, hypertrophy of some surrounding cardiomyocytes, atrophy of some cardiomyocytes, and scattered fibrous tissue hyperplasia forming scar foci. Brain tissue edema, nerve cells swelling degeneration, no other serious pathological changes. Oil red staining of frozen sections of brain tissue shows orange red lipid droplets in some small blood vessels. Liver tissue mild fatty liver. No obvious pathological changes were observed in other tissues under the microscope.

Forensic pathology diagnosis: 1. Pulmonary fat embolism with diffuse small vessel thrombosis. Acute pulmonary edema; 2. Cerebral fat embolism; Brain edema; 3. Atherosclerotic heart disease, grade II anterior descending coronary artery stenosis; 4. Infiltration of adipose tissue from sinoatrial node and atrioventricular node accompanied by formation of focal myocardial fibroid lesions; 5. Fatty liver (mild); 6. Multiple rib fractures and sternum fractures (caused by cardiopulmonary resuscitation) on both sides of the chest.

\section{Discussions}

In clinical and forensic studies, FES deaths caused by spinal surgery, such as vertebral fusion and vertebroplasty, are relatively rare. There are only a few case reports ${ }^{[2][3][4][5][6][7][8]}$ in the existing domestic and foreign clinical medical literature. This patients, with preoperative clinical examination, demonstrated no serious trauma history in hyperlipidemia and limbs, autopsy can see liver mild fatty liver lesions, the lack of other vital organs lethality based disease pathology change, therefore, can be ruled out because of severe trauma, hyperlipidemia and its basic diseases caused by the possibility of fat embolism or sudden death. Combined with the clinical characteristics of sudden change of condition to death during lumbar spine surgery and the findings of autopsy pathological tissue, the characteristics of death caused by intra-operative FES can be determined.

\subsection{Clinical morbidity and mortality of FES}

FES may be induced by the presence of fat emboli or free lipid acids in the lung or circulatory system. Since the first case of fat embolism syndrome was described by Zenker ${ }^{[9]}$ in 1862, cases of fat embolism have been reported and brought to clinical attention. However, the reported incidence of FES varies widely ( $1 \%$ to $29 \%$ ) due to a number of factors, but is generally related to the severity of the trauma and the number of long tubular bone fractures. FES is less common in upper limb fractures. Investigation shows that the incidence of pulmonary fat embolism after fracture is $7 \%$, but the fatality rate of patients with severe bone fracture injury and postoperative infection is as high as $62 \%{ }^{[10][11]}$.

Existing medical studies show that with the increase of various accidents and medical disputes, the incidence of FES has increased significantly. Patients with severe trauma, especially multiple fractures (such as femur, tibia, or pelvis) with hypovolemic shock, are at higher risk of FES, and the risk of closed fractures is higher than that of open fractures. Bone and joint replacements (such as intramedullary nailing, hip and knee replacement) are more common in nontraumatic patients, and FES is increasingly common in spinal arthrodesis. In addition, other medical-related cases, such as liposuction and fat transplantation in cosmetic surgery, cardiopulmonary bypass, pancreatitis, joint repair, severe burns, sickle cell anemia, and intravenous lipid transfusion, can also cause fat embolism syndrome ${ }^{[12][13]}$. Recent studies have also revealed that autologous blood transfusion during orthopedic surgery and cardiopulmonary resuscitation (with rib fractures) can lead to fat embolism ${ }^{[14][15][16][17]}$. 


\subsection{Mechanism of FSE injury}

FES is a complex process, and the specific mechanism is still controversial. At present, the main debate is around mechanical theory and biochemical theory, but it may also be that the two theories work together to lead to a series of pathophysiological changes ${ }^{[18]}$. The mechanical theory holds that fatty particles from bone marrow or adipose tissue enter the venous circulation under the action of external forces such as trauma or invasive surgery. Under the interaction between the stress response of the body and the fat particles in the blood after trauma, the blood rheology of the body changes and the coagulation function is abnormal, and further interacts with the fat particles in the blood to increase its volume. When passing through pulmonary microvasculature, embolism can easily occur, resulting in pulmonary dysfunction, and can also block microvasculature of other organ systems, resulting in multiple organ dysfunction. Biochemical theory suggests that although FES often occurs after trauma, it has also been reported to occur under non-traumatic conditions, suggesting that biochemical reactions in addition to mechanical processes play an important role in FES. When fat emboli enter the venous circulation, they will cause inflammation and abnormal coagulation function, rapidly produce fibrin and platelet aggregation, and further promote the production and release of free fatty acids into the blood. Under the action of inflammatory factors, free fatty acids aggregate into fat droplets and enter the microvascular system of tissues and organs with blood circulation, resulting in a series of clinical symptoms such as ARDS, myocardial systolic dysfunction, multi-organ dysfunction and failure. Comprehensive theory theory thinks, adipose embolus produces mechanical obstruction in pulmonary blood vessel, stimulate peripheral vascular endothelial cell to release a large number of lipase or because of the body stress response after trauma, induce adrenal gland to secrete catecholamine. Catecholamines not only mobilize lipids, raise blood lipids, but also activate adenine cyclase, thereby catalyzing inactive lipase in serum into active lipase. Under the action of lipase, neutral fat is hydrolyzed into free fatty acids and glycerin, and the blocked pulmonary blood vessels are stimulated by free fatty acids, resulting in chemical vascular inflammation, further aggravating the injury and presenting a series of clinical symptoms.

Based on the existing clinical reports, the sources of adipose particles in FES of lumbar fusion may include: (1) the injured adipose tissue at the surgical site; (2) Autologous blood transfusion after massive intraoperative bleeding; (3) Free lipid droplets from intra-operative cardiopulmonary resuscitation of rib fractures. Pathological examination of this case indicated that although the number and area of lipid droplets in the lung tissue during lumbar fusion were not extensive or large, they caused obvious chemical inflammatory reaction, and a large amount of inflammatory leukocytes, cellulose and platelets gathered around the lipid droplets and formed extensive small intravascular microthrombus. Accordingly, this case more confirmed the comprehensive theory.

\subsection{Clinical diagnostic criteria and pathological grading}

Up to now, there is still a lack of unified diagnostic criteria for FES in clinical medicine. In medical practice, a comprehensive judgment can only be made based on clinical manifestations, imaging data and susceptibility factors. Therefore, many experts have proposed clinical diagnostic criteria for FES, but the diagnostic criteria first proposed by Gurd are most commonly used at present ${ }^{[19]}$. Diagnosis criteria are: (1) more than 2 major indicators; (2) 1 main index +2 secondary indexes; (3) 1 main indicator + more than 4 reference indicators. Suspected criteria: 1 secondary index + more than 4 reference indexes. Lindeque et al. applied the results of blood gas analysis to the diagnosis of FES in patients with tibia and/or femur fractures on the basis of Gurd, and FES can be diagnosed as long as there is one or more indicators. Schonfeld et al. further quantified the related indicators and assigned different scores to each indicator. FES could be diagnosed with a total score $\geqslant 5$ (see table). Professor Wang Yickeng further summarized and proposed FES reference diagnostic criteria based on Gurd ${ }^{[20]}$. Early diagnosis: (1) History of fracture trauma; (2) Significant hypoxemia (arterial partial pressure of oxygen $<70 \mathrm{mmHg}$ after age correction), which cannot be explained by other reasons; (3) Although there were no major indicators of Gurd, there were corresponding reference indicators (serum lipase increase, serum free fatty acid increase to anemia and fundus changes in item 6 and 7). Clinical diagnosis: (1) History of fracture trauma; (2) Clear incubation period; (3) Significant or severe hypoxemia; (4) One or more major indicators of gurd and related reference indicators (anemia and fundus changes instead of items 6 and 7).

The above diagnostic criteria for FES are still made based on the relevant examination results and clinical manifestations during the clinical treatment during hospitalization. FES associated with spinal fusion have unique clinical characteristics, namely: early attack of the disease in a short time without incubation or short incubation period. Consequently, there lacks adequate time for imaging examination and clinical examination. Intraoperative blood gas analysis and electrocardiogram examination were the main auxiliary diagnostic bases, and the diagnostic criteria were mainly based on the exclusion of other diseases. Based on the characteristics of this case and other patients with fusion-related fat embolism, we propose diagnostic criteria for fusion-related fat embolism: (1) A history of fusion-related surgery, especially lower lumbar spine surgery; (2) It can occur during spinal fusion surgery or within 24 hours after surgery; (3) Patients are obese, especially those with thick 
adipose tissue in the lower back and buttocks; (4) More acute intraoperative bleeding $(\mathrm{Hb}<80 \mathrm{~g} / \mathrm{L})$; or with a history of intraoperative autologous blood transfusion; (5) Blood oxygen saturation decreased gradually during the operation, and blood gas analysis showed hypoxemia $(\mathrm{PO}<70 \mathrm{mmHg})$ or hyperco2 $\left(\mathrm{PCO}_{2}>50 \mathrm{mmHg}\right)$; (6) Acute myocardial infarction and acute cerebral apoplexy can be excluded if consciousness disturbance occurs; (7) The residual effect of postoperative muscle relaxants was eliminated.

Pathological diagnosis of fat embolism is the most effective method for diagnosis. In conventional HE staining sections, beaded and bright vacuolar changes can be seen in the capillaries or small vessels in the alveolar septum, which can be used as evidence for preliminary diagnosis of fat embolism. The use of special stains such as Sudan III staining can be further confirmed.

In terms of pathologic evaluation of the severity of fat embolism, endoscopic fat embolism in lung tissue was divided into five grades ${ }^{[21]}$ : (1) Grade 0: no fat droplets were observed in lung tissue under low magnification; (2) Grade $i$ : $1 \sim 5$ lipid droplets were observed under 5× microscope; (3) Grade ii : more than 5 lipid droplets were observed under 5× microscope; (4) Grade iii : $1 \sim 5$ lipid droplets were observed under 10× microscope; (5) Grade iv : $20 \times$ More than 5 lipid droplets were observed under microscope. According to their study, among the 11 cases of fat embolism below grade ii, except for 1 case as the auxiliary cause of death of pulmonary thromboembolism, the remaining 10 cases were only detected fat droplets in the lung tissue. Therefore, it was considered that fat embolism below grade ii could not cause the occurrence of FES in general and did not constitute the cause of death. Among the 16 cases of grade ii and ii $\sim$ iii fat embolism, 6 cases $(38 \%)$ were the direct cause of death, and the other 10 cases were the combined or auxiliary cause of death. Therefore, grade ii and ii $\sim$ iii fat embolism could cause FES, but the degree was relatively mild, and could be the cause of death alone. But death is more likely to occur when other factors are involved. Among 25 cases with grade iii or above, FES was the direct cause of death in 24 cases (96\%), except 1 case of craniocerebral trauma as absolute fatal injury. In addition, 8 cases of grade iii $\sim$ iv and grade iv fat embolism were associated with cerebral and/or renal fat embolism. Therefore, grade iii and above fat embolism could be considered as absolutely fatal injury. Samdanci et al. ${ }^{[14]}$ also used similar histopathological methods to study the histology of microscopic fat embolism. They classified the histology of microscopic fat embolism into: (1) Grade I: no fat droplets were observed at low magnification (4×); (2) Grade II: 2 5 lipid droplets were observed under 10× microscope; (3) Grade III: 40× 1 5 lipid droplets under microscope; (4) Grade IV: 40× More than 5 lipid droplets were observed under the microscope. The results showed that the pathological grade of trauma patients was significantly higher than that of nontrauma group. The pathological grading of the patients undergoing CPR with rib fracture was significantly higher than that of the patients without rib fracture. But they also stressed that broken ribs during CPR could not be considered medical error.

\section{Clinical treatment and suggestions}

At present, there is still no specific treatment for FES in clinical medicine, and there are different controversial views on relevant treatment schemes. The anticoagulant drugs heparin and corticosteroids are commonly used in medical practice, but there is no evidence that they improve morbidity or mortality. At present, systematic anticoagulation is the more common treatment. Heparin effectively stimulates lipid activity, thereby accelerating lipid clearance in circulation. In addition, anticoagulant therapy is not conducive to wound repair and pre-existing hematologic abnormalities, and there is no trial evidence to support the routine use of heparin and other anticoagulants for FES. However, in the case of FES after spinal fusion, based on the findings of postmortem pathological examination, it suggests that there are still appropriate indications for strengthening the use of anticoagulants and glucocorticoids. At the same time, in the rescue process, it is necessary to avoid the use of autologous blood transfusion and reduce the excessive injury to the ribs caused by chest compressions. Medical institutions with adequate material conditions are recommended to use mechanical life support equipment and extracorporeal membrane oxygenator, which is expected to improve the probability of successful rescue.

In light of the rescue treatment, it is more important to strengthen corresponding prevention before spinal fusion, and possible specific prevention measures include: (1) Strengthening preoperative risk assessment, such as high coagulation state and based disease in patients with risk factors assessment, assessment and evaluation, the complexity of spinal fusion zone of adipose tissue thickness and blood loss assessment, etc.; (2) Reducing and alleviating the contusion of adipose tissue, and reducing bleeding amount in the operation area; (3) Preparing enough blood for high-risk patients and minimize the use of autologous blood transfusion; (4) Strengthening observation of intraoperative anesthesia, especially for blood oxygen saturation monitoring and blood gas analysis, to early detection of respiratory abnormalities and make appropriate treatment measures; (5) Early identification and diagnosis of FE should be made according to respiratory abnormalities during intraoperative and early postoperation, and comprehensive treatment such as anticoagulation should be performed with low molecular weight heparin. 


\section{Conclusion}

FES is relatively common with high fatality rate in clinical practice. In addition to the common causes of fat embolism, attention should be paid to FES pertinent to spinal fusion in clinical practice. In addition, fat embolism can also be caused by special causes such as short and bony fractures of hands and feet, non-blunt violence injury, cosmetic surgery such as liposuction and fat transplantation, extremely refractory non-convulsive persistent epilepsy, firearm injury, and renal angioleiomyoma. At present, the mechanism of fat embolism is not completely clear, the clinical manifestations of early fat embolism patients are not obvious, the diagnosis proves to be with more difficulties. Delaying treatment of fat embolism syndrome increases the risk of death. The purpose of this paper is to explore the clinical characteristics, diagnostic criteria and pathological characteristics of FES related to spinal fusion, so as to provide reference suggestions for the clinical diagnosis and treatment of similar cases in the future, and to provide relevant medical perspectives for the prudent and reasonable cause of death inference and diagnosis and treatment behavior fault judgment in judicial identification work.

\begin{tabular}{|c|c|c|c|}
\hline \multicolumn{4}{|c|}{ Criteria for the Diagnosis of Fat Embolism Syndrome } \\
\hline Criteria & \multicolumn{2}{|c|}{ Findings } & \\
\hline \multirow[t]{13}{*}{ Gurd Criteria } & \multicolumn{3}{|l|}{ Major } \\
\hline & \multicolumn{3}{|l|}{ Petechial rash } \\
\hline & \multicolumn{3}{|c|}{ Respiratory symptoms with radiographic changes } \\
\hline & \multicolumn{3}{|c|}{ Central nervous system signs unrelated to trauma or other condition } \\
\hline & \multicolumn{3}{|l|}{ Minor } \\
\hline & Tachycardia & \multicolumn{2}{|l|}{$(>110 \mathrm{bpm})$} \\
\hline & Pyrexia & \multicolumn{2}{|l|}{$\left(>38.5^{\circ} \mathrm{C}\right)$} \\
\hline & Retinal changes & \multicolumn{2}{|l|}{ (fat or petechiae) } \\
\hline & Renal changes & \multicolumn{2}{|c|}{ (oliguria, anuria, or lipiduria) } \\
\hline & \multicolumn{3}{|c|}{ Acute decrease in hemoglobin } \\
\hline & \multicolumn{3}{|c|}{ Acute thrombocytopenia } \\
\hline & \multicolumn{3}{|c|}{ Increasing ESR } \\
\hline & \multirow{2}{*}{\multicolumn{3}{|c|}{ Fat globules in sputum }} \\
\hline \multicolumn{2}{|c|}{ Schonfeld Criteria ${ }^{b}$} & & \\
\hline & \multirow{2}{*}{\multicolumn{2}{|c|}{$\mathrm{X}$-ray chest diffuss infiltrates }} & (5 point) \\
\hline & & & (4 point) \\
\hline & Hypoxemia & $\left(\mathrm{PaO}_{2}<9.3 \mathrm{kpa}\right)$ & (3 point) \\
\hline & Fever & $\left(>38^{\circ} \mathrm{C}\right)$ & (1 point) \\
\hline & Tachycardia & $(>120 \mathrm{bpm})$ & (1 point) \\
\hline & Tachypnea & (>30bpm) & (1 point) \\
\hline & Confusion & & (1 point) \\
\hline \multicolumn{4}{|c|}{ Lindeque Criteria ${ }^{\mathrm{c}}$} \\
\hline & \multicolumn{3}{|c|}{ Sustained $\mathrm{PaO} 2<8$ kpa } \\
\hline & \multicolumn{3}{|c|}{ Sustained $\mathrm{PCO} 2>7.3 \mathrm{kpa}$ orPH $<7.3$} \\
\hline & \multirow{2}{*}{\multicolumn{3}{|c|}{$\begin{array}{l}\text { Sustained respirtory rate }>35 \mathrm{bpm} \text {, in spite of sedation } \\
\text { Increased work of breathing, dyspnea, tachycardia, anxiety }\end{array}$}} \\
\hline & & & \\
\hline
\end{tabular}

\section{References}

[1] Milroy CM, Parai JL. Fat Embolism, Fat Embolism Syndrome and the Autopsy. Academic Forensic Pathology. 2019, 9(3-4): 136-154.

[2] J E Gittman, T A Buchanan, B J Fisher, P S Bergeson, P E Palmer. Fatal fat embolism after spinal fusion for scoliosis. JAMA. 1983, 249(6): 779-81.

[3] Brandt SE, Zeegers WS, Ceelen TL. Eur Spine J. Fatal pulmonary fat embolism after dorsal spinal fusion. European Spine Journal. 1998, 7(5): 426-8.

[4] Stroud MH, McCarthy RE, Parham DM, Schexnayder SM. Fatal pulmonary fat embolism following spinal fusion sur- 
gery. Pediatr Crit Care Med. 2006, 7(3): 263-6.

[5] Meng XL, Zhao H, Su QJ, Hai Y. Acute pulmonary embolism following adolescent idiopathic scoliosis correction surgery: case report and review of literature. J Int Med Res. 2013, 41(5): 1759-67.

[6] Michael H Stroud, Richard E McCarthy, David M Parham, Stephen M Schexnayder. Fatal pulmonary fat embolism following spinal fusion surgery. Pediatr Crit Care Med. 2006, 7(3): 263-6.

[7] Yoshimasa Takahashi, Ken'ichiro, Narusawa, Kenji Shimizu, Masakazu Takata. Fatal pulmonary fat embolism after posterior spinal fusion surgery. Journal of Orthopaedic Science. 2006, 11(2): 217-20.

[8] Zaihua Zhou, Jianye Liu. A case of fat embolism syndrome after lumbar tuberculosis surgery. China Journal of Orthopaedics and Traumatology. 2002, 15(10): 625.

[9] Christopher M. Milroy, Jacqueline L. Parai. Fat Embolism, Fat Embolism Syndrome and the Autopsy. Acad Forensic Pathol. 2019, 9(3-4): 136-154.

[10] Wang Huasong, Cai Xianhua, Liu Ximing et al. Clinical analysis of recurrence of fractures with fat embolism syndrome after internal fixation. Chinese Journal of Orthopaedic Trauma. 2013, 15 (6): 505-508.

[11] Li S, Zou D, Qin Z, et al. Nonfracture-associated pulmonary fat embolism after blunt force fatality: case report and review of the literature. Am J Forensic Med Pathol. 2015, 36(2): 61-66.

[12] Miller, Peter, Prahlow, Joseph A. Autopsy Diagnosis of Fat Embolism Syndrome. American J Forensic Med Pathol. 2011, 32 (3): 291-299.

[13] Lauren E Fukumoto, Kathryn D Fukumoto. Fat Embolism Syndrome. Nurs Clin North Am. 2018, 53(3): $335-347$.

[14] Emine Turkmen Samdanci, Muhammet Reha Celik, Sultan Pehlivan, Osman Celbis, Dilhan Turkkan, Dogus Ozdemir Kara, Esra Pamukcu. Histopathological evaluation of autopsy cases with isolated pulmonary fat embolism (IPFE): is cardio -pulmonary resuscitation a main cause of death in IPFE? Open Access Emerg Med. 2019, 11: 121-127.

[15] Ondruschka B, Baier C, Bernhard M, Buschmann C, Dreßler J, Schlote J, Zwirner J, Hammer N. Frequency and intensity of pulmonary bone marrow and fat embolism due to manual or automated chest compressions during cardiopulmonary resuscitation. Forensic Sci Med Pathol. 2019, 15(1): 48-55.

[16] Chen Haofei, Sun Hong. A case of fat embolism syndrome caused by autologous blood transfusion during spinal surgery. Psychological Doctor. 2011, 9: 1339.

[17] Magnus Jacobsson, Anders Bengtsson. Fat embolism and autologous blood transfusions in orthopaedic surgery. Current Anaesthesia \& Critical Care. 2004, 15(2): 87-93.

[18] David L. Rothberg, Christopher A. Makarewich. Fat embolism and fat embolism syndrome. J Am Acad Orthop Surg. 2019, 27(8): e346-e355.

[19] Gurd AR. Fat embolism: an aid to diagnosis. J Bone Joint Surg Br. 1970, 52: 732-737.

[20] Wang Yiceng, Meng Jimao, Guo Ziheng. Bone and joint Injury[M]. Beijing: People's Medical Publishing House, 1990: 241-247.

[21] Dou Guoyan, Fan Qinhe fan, et al. The relationship between traumatic death and pathological grade of pulmonary fat embolism in elderly patients. Practical Geriatrics. 2017, 31(7): 643-645.

[22] Timon C, Keady C, Murphy CG. Fat Embolism Syndrome - A Qualitative Review of its Incidence, Presentation, Pathogenesis and Management. Malaysian Orthopaedic Journal. 2021,15(1): 1-11. 